\title{
Two-part set systems
}

\author{
Péter L. Erdős ${ }^{\mathrm{a}, *} \quad$ Dániel Gerbner ${ }^{\mathrm{a}, \dagger} \quad$ Nathan Lemons $^{\mathrm{a}, \mathrm{b}, \uparrow}$ \\ Dhruv Mubayi ${ }^{\mathrm{c}, \ddagger} \quad$ Cory Palmer ${ }^{\mathrm{a}, \mathrm{d}, \dagger} \quad$ Balázs Patkós ${ }^{\mathrm{a}, \S}$
}

\author{
${ }^{a}$ Hungarian Academy of Sciences, Alfréd Rényi Institute of Mathematics, \\ P.O.B. 127, Budapest H-1364, Hungary \\ ${ }^{\mathrm{b}}$ Theoretical Division, Los Alamos National Laboratory, Los Alamos, NM 87545, USA \\ ${ }^{\mathrm{c}}$ University of Illinois at Chicago, Chicago, IL 6060\%, US \\ ${ }^{\mathrm{d}}$ University of Illinois at Urbana-Champaign, Urbana, IL 61801, US
}

Submitted: Oct 1, 2011; Accepted: Feb 21, 2012; Published: Mar 9, 2012

Mathematics Subject Classification: 05D05

\begin{abstract}
The two part Sperner theorem of Katona and Kleitman states that if $X$ is an $n$-element set with partition $X_{1} \cup X_{2}$, and $\mathcal{F}$ is a family of subsets of $X$ such that no two sets $A, B \in \mathcal{F}$ satisfy $A \subset B$ (or $B \subset A$ ) and $A \cap X_{i}=B \cap X_{i}$ for some $i$, then $|\mathcal{F}| \leq\left(\begin{array}{c}n \\ n / 2 \mid\end{array}\right)$. We consider variations of this problem by replacing the Sperner property with the intersection property and considering families that satisfy various combinations of these properties on one or both parts $X_{1}, X_{2}$. Along the way, we prove the following new result which may be of independent interest: let $\mathcal{F}, \mathcal{G}$ be intersecting families of subsets of an $n$-element set that are additionally crossSperner, meaning that if $A \in \mathcal{F}$ and $B \in \mathcal{G}$, then $A \not \subset B$ and $B \not \subset A$. Then $|\mathcal{F}|+|\mathcal{G}| \leq 2^{n-1}$ and there are exponentially many examples showing that this bound is tight.
\end{abstract}

Keywords: extremal set theory, Sperner, intersecting

\footnotetext{
* Research supported in part by the Hungarian NSF, under contract NK 78439 and K 68262 . email: erdos.peter@renyi.mta.hu

${ }^{\dagger}$ Research supported in part by the Hungarian NSF, under contract NK 78439 . email: gerbner.daniel@renyi.mta.hu, lemons.nathan@renyi.mta.hu, ctpalmer@illinois.edu

${ }_{\ddagger}^{\ddagger}$ Research supported in part by NSF grant DMS-0969092. email: mubayi@math.uic.edu

$\S$ Research supported by Hungarian NSF, under contract PD-83586, and the János Bolyai Research Scholarship of the Hungarian Academy of Sciences.

email: patkos.balazs@renyi.mta.hu
} 


\section{Introduction}

Let $X$ be a finite set and let $2^{X}$ be the system of all subsets of $X$. The basic problem in extremal set theory is to determine the maximum size that a set system $\mathcal{F} \subseteq 2^{X}$ can have provided $\mathcal{F}$ satisfies a prescribed property. The prototypes of investigated properties are the intersecting and Sperner properties. A set system $\mathcal{F}$ is intersecting if $F_{1} \cap F_{2} \neq \emptyset$ for any pair $F_{1}, F_{2} \in \mathcal{F}$ and a set system $\mathcal{F}$ is Sperner if there do not exist two distinct sets $F_{1}, F_{2} \in \mathcal{F}$ such that $F_{1} \subset F_{2}$. The celebrated theorems of Erdös, Ko, Rado [4] and of Sperner [13] determine the largest size that a uniform intersecting set system and Sperner system can have. Both theorems have many applications and generalizations.

One such generalization of the Sperner property is the so called more part Sperner property. In this case, the underlying set $X$ is partitioned into $m$ subsets $X_{1}, \ldots, X_{m}$ and the system $\mathcal{F} \subset 2^{X}$ is said to be $m$-part Sperner if for any pair $F_{1}, F_{2} \in \mathcal{F}$ with $F_{1} \subset F_{2}$ there exist at least two indices $1 \leq i_{1}<i_{2} \leq m$ such that $F_{1} \cap X_{i_{j}} \neq F_{2} \cap X_{i_{j}}$ holds for $j=1,2$. Systems with this property were first considered in $[9,11]$; for a survey of recent results see [2].

In this paper we will consider analogous problems for intersection properties and also some mixed more part properties in the case when $m$ equals 2. All maximum size 2part Sperner set systems were described by P.L. Erdős and G.O.H. Katona in [5, 6]. To rephrase the 2-part Sperner property it is convenient to introduce the following set systems of traces: for any $A \subseteq X_{1}$ and $B \subseteq X_{2}$ let $\mathcal{F}_{A}=\left\{F \cap X_{2}: F \in \mathcal{F}, F \cap X_{1}=\right.$ $A\}, \mathcal{F}_{B}=\left\{F \cap X_{1}: F \in \mathcal{F}, F \cap X_{2}=B\right\}$. Also, for any $F \in \mathcal{F}$ we will call $F \cap X_{1}$ and $F \cap X_{2}$ the traces of $F$ on $X_{1}$ and $X_{2}$. One can easily see that a set system $\mathcal{F}$ is 2-part Sperner with respect to the partition $X=X_{1} \cup X_{2}$ if and only if for any subset $A \subseteq X_{1}$ or $B \subseteq X_{2}$ the set systems $\mathcal{F}_{A}$ and $\mathcal{F}_{B}$ possess the Sperner property.

Having this equivalence in mind, it is natural to introduce the following three definitions where we always assume that the underlying set $X$ is partitioned into two sets $X_{1}$ and $X_{2}$ :

Definition 1. (i) a set system $\mathcal{F} \subseteq 2^{X}$ is 2-part intersecting (a 2I-system for short) if for any subset $A$ of $X_{1}$ (and for any subset $B$ of $X_{2}$ ) the trace system $\mathcal{F}_{A}$ on $X_{2}$ (and the trace system $\mathcal{F}_{B}$ on $X_{1}$ ) is intersecting,

(ii) a set system $\mathcal{F} \subseteq 2^{X}$ is 2-part intersecting, 2-part Sperner (a 2I2S-system for short) if for any subset $A$ of $X_{1}$ and for any subset $B$ of $X_{2}$ the trace systems $\mathcal{F}_{A}$ on $X_{2}$ and $\mathcal{F}_{B}$ on $X_{1}$ are intersecting and Sperner,

(iii) a set system $\mathcal{F} \subseteq 2^{X}$ is 1-part intersecting, 1-part Sperner (a 1I1S-system for short) if there exists no pair of distinct sets $F_{1}, F_{2}$ in $\mathcal{F}$ such that the traces of $F_{1}, F_{2}$ are disjoint at one of the parts and are in containment at the other.

We will address the problem of finding the maximum possible size of a set system possessing the properties above. Some of our bounds will apply regardless of the sizes of the parts in the 2-partition and some will only apply to special cases. We will be mostly interested in the case when $\left|X_{1}\right|=\left|X_{2}\right|$. Clearly, for any 2-part set system $\mathcal{F}$ we have $|\mathcal{F}|=\sum_{A \subseteq X_{1}}\left|\mathcal{F}_{A}\right|=\sum_{B \subseteq X_{2}}\left|\mathcal{F}_{B}\right|$. As any intersecting system of subsets of $X_{1}$ has size 
at most $2^{\left|X_{1}\right|-1}$, it follows that any 2 I-system has size at most $2^{\left|X_{2}\right|} 2^{\left|X_{1}\right|-1}=2^{|X|-1}$. In Section 2 we will prove the following theorem.

Theorem 2. Let $\mathcal{F} \subseteq 2^{X}$ be a 2-part intersecting system of maximum size. If the 2partition $X=X_{1} \cup X_{2}$ is non-trivial (i.e. $X_{1} \neq \emptyset, X_{2} \neq \emptyset$ ), then the following inequality holds:

$$
|\mathcal{F}| \leq \frac{3}{8} 2^{|X|}
$$

The bound is best possible if $X_{1}$ or $X_{2}$ is a singleton. Moreover, if $\left|X_{1}\right|=\left|X_{2}\right|$, then there exists a 2-part intersecting system of size $\frac{1}{3}\left(2^{|X|}+2\right)$.

The rest of Section 2 is devoted to 2I2S systems. We prove the following result.

Theorem 3. Let $\mathcal{F} \subseteq 2^{X}$ be a 2-part intersecting, 2-part Sperner system of maximum size. Then $|\mathcal{F}| \leq\left(\begin{array}{c}|X| \\ \lceil|X| / 2\rceil\end{array}\right)$ holds. This bound is asymptotically sharp as long as $\left|X_{1}\right|=$ $o\left(\left|X_{2}\right|^{1 / 2}\right)$. If $\left|X_{1}\right|=\left|X_{2}\right|$ holds, then there exists a 2I2S system of size $c\left(\begin{array}{c}|X| \\ {[|X| / 2\rceil}\end{array}\right)$ with $c>2 / 3$.

The main result of the paper is proved in Section 3. We determine the maximum size of a 1-part intersecting 1-part Sperner set system.

Theorem 4. Let $\mathcal{F}$ be a maximum size 1-part intersecting, 1-part Sperner set system. Then $|\mathcal{F}|=2^{|X|-2}$.

\section{2I- and 2I2S-systems}

In this section we consider two-part intersecting and two-part intersecting, two-part Sperner set systems. We first consider a general construction that produces large families with these properties. Let $\mathcal{A}_{1}, \ldots, \mathcal{A}_{m}$ and $\mathcal{B}_{1}, \ldots, \mathcal{B}_{m}$ be partitions of $2^{X_{1}}$ and $2^{X_{2}}$ into disjoint intersecting (or intersecting, Sperner) systems some of which may possibly be empty. Then the set system $\mathcal{F}:=\cup_{i=1}^{m} \mathcal{A}_{i} \times \mathcal{B}_{i}=\left\{A \cup B: A \in \mathcal{A}_{i}, B \in \mathcal{B}_{i}\right.$ for some $\left.1 \leq i \leq m\right\}$ is a $2 \mathrm{I}-(2 \mathrm{I} 2 \mathrm{~S})$-system by definition.

Fact 5. Let $0 \leq x_{1} \leq \cdots \leq x_{n}, 0 \leq y_{1} \leq \cdots \leq y_{n}$ be real numbers and $\pi$ be a permutation of the first $n$ integers. Then we have the following inequalities:

$$
\sum_{i=1}^{n} x_{i} y_{\pi(i)} \leq \sum_{i=1}^{n} x_{i} y_{i} \leq \max \left\{\sum_{i=1}^{n} x_{i}^{2}, \sum_{i=1}^{n} y_{i}^{2}\right\}
$$

Thus to maximize the size of a family obtained through the general construction one should enumerate the $\mathcal{A}_{i}$ 's and the $\mathcal{B}_{i}$ 's in decreasing order according to their size. Moreover, if $\left|X_{1}\right|=\left|X_{2}\right|$, then it is enough to consider partitions $\mathcal{A}_{1}, \ldots, \mathcal{A}_{m}$ of $2^{X_{1}}$ and the $\operatorname{sum} \sum_{i=1}^{m}\left|\mathcal{A}_{i}\right|^{2}$. 


\subsection{Two-part intersecting systems}

In this subsection we prove Theorem 2. In the proof we use the following theorem of Kleitman [10].

Theorem 6 (Kleitman [10]). Let $\mathcal{F}_{1}, \ldots, \mathcal{F}_{m} \subseteq 2^{[n]}$ be intersecting set systems. Then

$$
\left|\mathcal{F}_{1} \cup \cdots \cup \mathcal{F}_{m}\right| \leq 2^{n}-2^{n-m} .
$$

Proof of Theorem 2. For any subset $A$ of $X_{1}$ let $\bar{A}$ denote its complement $X_{1} \backslash A$. By definition, both $\mathcal{F}_{A}$ and $\mathcal{F}_{\bar{A}}$ are intersecting. Also, these set systems are disjoint as $B \in \mathcal{F}_{A} \cap \mathcal{F}_{\bar{A}}$ implies $B \cup A, B \cup \bar{A} \in \mathcal{F}$ which contradicts the 2-part intersecting property of $\mathcal{F}$. Thus by Theorem 6 we have $\left|\mathcal{F}_{A}\right|+\left|\mathcal{F}_{\bar{A}}\right| \leq 2^{\left|X_{2}\right|-1}+2^{\left|X_{2}\right|-2}$.

Altogether we obtain

$$
|\mathcal{F}| \leq 2^{\left|X_{1}\right|-1}\left(2^{\left|X_{2}\right|-1}+2^{\left|X_{2}\right|-2}\right)=\frac{3}{8} 2^{|X|} .
$$

Our best lower bounds arise from our general construction. If $X_{1}$ consists of a single element $x_{1}$, then let $\mathcal{A}_{1}=\left\{\left\{x_{1}\right\}\right\}, \mathcal{A}_{2}=\{\emptyset\}$ and $\mathcal{B}_{1}=\left\{B \subset X_{2}: x_{2} \in B\right\}, \mathcal{B}_{2}=$ $\left\{B \subset X_{2}: x_{2} \notin B, x_{2}^{\prime} \in B\right\}$ for two fixed elements $x_{2}, x_{2}^{\prime} \in X_{2}$ and the other $\mathcal{B}_{i}$ 's be arbitrary while the other $\mathcal{A}_{i}$ 's be empty. For the set system $\mathcal{F}$ we obtain via the general construction, we have $|\mathcal{F}|=2^{\left|X_{2}\right|-1}+2^{\left|X_{2}\right|-2}=\frac{3}{8} 2^{|X|}$.

Finally, let us suppose that $\left|X_{1}\right|=\left|X_{2}\right|=|X| / 2$ and let the elements of $X_{1}$ and $X_{2}$ be $x_{1}^{1}, \ldots, x_{m}^{1}$ and $x_{1}^{2}, \ldots, x_{m}^{2}$. Let us define the partition of $2^{X_{1}}$ and $2^{X_{2}}$ in the following way: $\mathcal{A}_{i}:=\left\{A \subset X_{1} \backslash\left\{x_{1}^{1}, \ldots, x_{i-1}^{1}\right\}: x_{i}^{1} \in A\right\}, \mathcal{B}_{i}:=\left\{B \subset X_{2} \backslash\left\{x_{1}^{2}, \ldots, x_{i-1}^{2}\right\}: x_{i}^{2} \in B\right\}$ for all $1 \leq i \leq m+1$ (i.e. $\mathcal{A}_{m+1}=\mathcal{B}_{m+1}=\{\emptyset\}$ ). Then for the set system $\mathcal{F}$ arising from the general construction we have

$$
|\mathcal{F}|=1+\sum_{i=1}^{m} 2^{|X|-2 i}=\frac{2^{|X|}+2}{3} .
$$

Remark 7. Theorem 6 shows that the above set system for the $\left|X_{1}\right|=\left|X_{2}\right|$ case is best possible among those that we can obtain via the general construction. Indeed, by Fact 5 we know that we have to consider partitions of $2^{X_{1}}$ into intersecting set systems with sizes $s_{1}, s_{2}, \ldots, s_{m}$ and maximize $\sum_{i=1}^{m} s_{i}^{2}$. But a partition maximizes this sum of squares if for all $1 \leq j \leq m$ the sums $\sum_{i=1}^{j} s_{i}$ are maximized. In the construction we use, the sums $\sum_{i=1}^{j} s_{i}$ match the upper bound of Theorem 6 .

\subsection{Two-part intersecting, two-part Sperner systems}

In this subsection we consider 2I2S-systems and prove Theorem 3. To be able to use the general construction, we need to define a partition of the power set into intersecting Sperner set systems. 
Construction 8. Here we give a partition of the power set of $Y$ into intersecting Sperner systems where all levels are partitioned into minimal number of (uniform) intersecting systems (we call this canonical partition). This partition is in the form of

$$
\begin{aligned}
\mathcal{Y}_{k}, & \text { for } \quad k=\left\lceil\frac{|Y|+1}{2}\right\rceil, \ldots,|Y| \\
\mathcal{Y}_{i, j}, & \text { for } \quad i=1, \ldots,\left\lceil\frac{|Y|+1}{2}\right\rceil-1, j=1, \ldots,|Y|-2 i+1 ; \\
\mathcal{Y}_{\ell}^{*}, & \text { for } \quad \ell=0, \ldots,\left\lceil\frac{|Y|+1}{2}\right\rceil-1 .
\end{aligned}
$$

The systems $\mathcal{Y}_{k}$ are $\left(\begin{array}{l}Y \\ k\end{array}\right)$. Fix an enumeration $y_{1}, \ldots, y_{|Y|}$ of the elements of $Y$ and define the systems $\mathcal{Y}_{i, j}$ as $\left\{Y^{\prime} \in\left(\begin{array}{c}Y \backslash\left\{y_{1}, \ldots, y_{j-1}\right\} \\ i\end{array}\right): y_{j} \in Y^{\prime}\right\}$. Finally let $\mathcal{Y}_{\ell}^{*}=\left(\begin{array}{c}Y \\ \ell\end{array}\right) \backslash \bigcup_{j=1}^{|Y|-2 \ell+1} \mathcal{Y}_{\ell, j}$. We remark that the second and third types are identical to those in the corresponding Kneser construction. Note that the number of systems in the partition is quadratic in $|Y|$ but for any $\varepsilon>0$ there exists $K=K(\varepsilon)$ such that

$$
\left|\bigcup_{k=\left\lceil\frac{|Y|+1}{2}\right\rceil}^{|Y|} \mathcal{Y}_{k} \cup \bigcup_{i=|Y| / 2-K|Y|^{1 / 2}}^{|Y| / 2} \bigcup_{j=1}^{|Y|-2 i+1} \mathcal{Y}_{i, j} \cup \bigcup_{\ell=|Y| / 2-K|Y|^{1 / 2}}^{|Y| / 2} \mathcal{Y}_{\ell}^{*}\right| \geq(1-\varepsilon) 2^{|Y|} .
$$

Indeed, the sets in all the $\mathcal{Y}_{k}$ contain all subsets of $Y$ of size greater than $|Y| / 2$, and the remaining families $\mathcal{Y}_{i, j}, \mathcal{Y}_{\ell}^{*}$ contain all subsets of $Y$ of size between $|Y| / 2-K|Y|^{1 / 2}$ and $|Y| / 2$. Since the number of subsets of $Y$ of size less than $|Y| / 2-K|Y|^{1 / 2}$ is less than $\varepsilon 2^{|Y|}$, the inequality in (1) follows. It is easy to see that the number of set systems in the union in (1) is at most $2 K^{2}|Y|$.

Proof of Theorem 3. The upper bound of the theorem follows from the result of Katona [9] and Kleitman [11] stating that a 2-part Sperner system has size at most $\left(\begin{array}{c}|X| \\ \lceil|X| / 2\rceil\end{array}\right)$, since any 2I2S-system is 2-part Sperner.

We now prove the lower bound. For $i=1,2$ let $x_{i}=\left|X_{i}\right|$, and recall that $n=|X|=$ $x_{1}+x_{2}$. First we consider the case when the size of $x_{1}$ is negligible compared to the size of $x_{2}$. Let us assume that $x_{1}=o\left(x_{2}^{1 / 2}\right)$. As observed above, from the canonical partition of $2^{X_{1}}$ which has $\Theta\left(x_{1}^{2}\right)$ families, there are $m=O\left(x_{1}\right)$ families $\mathcal{F}_{1}^{1}, \ldots, \mathcal{F}_{m}^{1} \subset 2^{X_{1}}$ such that

$$
\left|\bigcup_{i=1}^{m} \mathcal{F}_{i}^{1}\right|=(1-o(1)) 2^{x_{1}}
$$

If $i=o\left(x_{2}^{1 / 2}\right)$ then the system $\left(\begin{array}{c}X_{2} \\ x_{2} / 2+i\end{array}\right)$ is intersecting Sperner and has size $(1-o(1))\left(\begin{array}{c}x_{2} \\ x_{2} / 2\end{array}\right)=$ $1 / 2^{x_{1}}(1-o(1))\left(\begin{array}{c}n \\ n / 2\end{array}\right)$. Thus, by the general construction, we obtain the following 2I2Ssystem from these partitions:

$$
\mathcal{F}=\bigcup_{i=1}^{m}\left\{F \cup H: F \in \mathcal{F}_{i}^{1}, H \in\left(\begin{array}{c}
X_{2} \\
x_{2} / 2+i
\end{array}\right)\right\} .
$$


By the above, $|\mathcal{F}|$ is equal to

$$
\sum_{i=1}^{m}\left|\mathcal{F}_{i}^{1}\right|\left(\begin{array}{c}
x_{2} \\
\frac{x_{2}}{2}+i
\end{array}\right) \geq \frac{1}{2^{x_{1}}}(1-o(1))\left(\begin{array}{c}
n \\
\frac{n}{2}
\end{array}\right) \sum_{i=1}^{m}\left|\mathcal{F}_{i}^{1}\right|=(1-o(1))\left(\begin{array}{c}
n \\
\frac{n}{2}
\end{array}\right) .
$$

Let us consider the case $x_{1}=x_{2}$. We first show that the 2I2S-system $\mathcal{F}$ we derive from the canonical partition using our general construction has size $(2 / 3-o(1))\left(\begin{array}{c}n \\ \lceil n / 2\rceil\end{array}\right)$. We then use Frankl and Füredi's construction [7] to improve this bound by a constant factor. For sake of simplicity, assume $n$ is divisible by 4 . Then our system has size

$$
\sum_{i=n / 4+1}^{n / 2}\left(\begin{array}{c}
n / 2 \\
i
\end{array}\right)^{2}+\sum_{i=1}^{n / 4} \sum_{k=0}^{n / 2-2 i}\left(\begin{array}{c}
n-1-k \\
i-1
\end{array}\right)^{2}+\sum_{i=1}^{n / 4}\left(\begin{array}{c}
2 i-1 \\
i
\end{array}\right)^{2}
$$

where the sums belong to the three different system types in the canonical partition. We can write our system as $\mathcal{F}=\mathcal{F}_{1} \cup \mathcal{F}_{2}$ where the first subsystem corresponds to the sets listed in the first summation, and the second one consists of the other sets. Then

$$
\begin{aligned}
\left|\mathcal{F}_{1}\right|=\sum_{i=n / 4+1}^{n / 2}\left(\begin{array}{c}
n / 2 \\
i
\end{array}\right)^{2} & =\sum_{i=n / 4+1}^{n / 2}\left(\begin{array}{c}
n / 2 \\
i
\end{array}\right)\left(\begin{array}{c}
n / 2 \\
n / 2-i
\end{array}\right) \\
& =1 / 2\left(\begin{array}{c}
n \\
n / 2
\end{array}\right)-\left(\begin{array}{c}
n / 2 \\
n / 4
\end{array}\right)^{2}=(1 / 2-o(1))\left(\begin{array}{c}
n \\
n / 2
\end{array}\right)
\end{aligned}
$$

as $\left(\begin{array}{c}n / 2 \\ i\end{array}\right)\left(\begin{array}{c}n / 2 \\ n / 2-i\end{array}\right)$ is the number of those $n / 2$-subsets of $X$ that intersect $X_{1}$ in $i$ elements.

Next we prove that $\left|\mathcal{F}_{2}\right| \geq(1 / 3-o(1))\left|\mathcal{F}_{1}\right|$ which implies that $\left|\mathcal{F}_{2}\right| \geq \frac{1 / 2-o(1)}{3}\left(\begin{array}{c}n \\ n / 2\end{array}\right)$ and thus $|\mathcal{F}| \geq\left(\frac{2}{3}-o(1)\left(\begin{array}{c}n \\ n / 2\end{array}\right)\right.$. We consider those members of $\mathcal{F}_{2}$ which intersect $X_{1}$ in $i$ elements (and then intersect $X_{2}$ in $i$ elements too). We will show that, for most values of $i$, the number of these sets is roughly a third of the number of those members of $\mathcal{F}_{1}$, which intersect $X_{1}$ (and then $X_{2}$ as well) in $n / 2-i$ elements. We have to compare

$$
S_{i}=\left(\begin{array}{c}
2 i-1 \\
i
\end{array}\right)^{2}+\sum_{k=0}^{n / 2-2 i}\left(\begin{array}{c}
n / 2-1-k \\
i-1
\end{array}\right)^{2} \text { to }\left(\begin{array}{c}
n / 2 \\
n / 2-i
\end{array}\right)^{2}=\left(\begin{array}{c}
n / 2 \\
i
\end{array}\right)^{2} .
$$

We will be done, if we establish $S_{i} /\left(\left(\begin{array}{c}n / 2 \\ i\end{array}\right)\right)^{2}=1 / 3+o(1)$ for all $n / 4-n^{2 / 3} \leq i \leq n / 4-\log n$ as

$$
\sum_{i<n / 4-n^{2 / 3}}\left(\begin{array}{c}
n / 2 \\
n / 2-i
\end{array}\right)^{2}+\sum_{n / 4-\log n<i \leq n / 2}\left(\begin{array}{c}
n / 2 \\
n / 2-i
\end{array}\right)^{2}=o\left(\left(\begin{array}{c}
n \\
n / 2
\end{array}\right)\right) .
$$

To deduce $S_{i} /\left(\left(\begin{array}{c}n / 2 \\ i\end{array}\right)\right)^{2}=1 / 3+o(1)$ we need the following fact.

Fact 9. Let $a_{1} \geq a_{2} \geq \cdots \geq a_{k}>0$ positive reals with $\sum_{\ell=1}^{k} a_{\ell}=1$. If for some $j<k$ we have $a_{\ell}=2^{-\ell}+o(1)$ for all $\ell<j$ and $\sum_{\ell=j}^{k} a_{\ell}=o(1)$, then $\sum_{\ell=1}^{k} a_{\ell}^{2}=1 / 3+o(1)$. 
All we have to do is to verify the conditions of Fact 9 to the numbers

$$
r_{\ell}=\frac{\left(\begin{array}{c}
n / 2-\ell \\
i-1
\end{array}\right)}{\left(\begin{array}{c}
n / 2 \\
i
\end{array}\right)} \text { for } \ell=1, \ldots, n / 2-2 i+1 \text { and } r_{n / 2-2 i+2}=\frac{\left(\begin{array}{c}
2 i-1 \\
i
\end{array}\right)}{\left(\begin{array}{c}
n / 2 \\
i
\end{array}\right)}
$$

with $j=\min \left\{n / 4-i, n^{1 / 4}\right\}$ and $k=n / 2-2 i+2$. First of all $\sum_{\ell} r_{\ell}=1$ as these numbers correspond to the ratios of set systems in a partition. Next we show that $r_{\ell}=2^{-\ell}+o(1)$ for all $\ell<j$. Writing $d_{\ell}=\frac{r_{\ell}}{r_{\ell-1}}$ for $2 \leq \ell \leq j-1$ and $i=n / 4-m$ we obtain

$$
d_{\ell}=\frac{r_{\ell}}{r_{\ell-1}}=\frac{\left(\begin{array}{c}
n / 2-\ell \\
i-1
\end{array}\right)}{\left(\begin{array}{c}
n / 2-\ell+1 \\
i-1
\end{array}\right)}=\frac{n / 2-\ell+i+2}{n / 2-\ell+1}=\frac{1}{2}+\frac{m-\ell / 2+3 / 2}{n / 2-\ell+1}=\frac{1}{2}+O\left(n^{-1 / 3}\right)
$$

and thus for $\ell<j \leq n^{1 / 4}$

$$
r_{1}=\frac{i}{n / 2}=\frac{1}{2}+o(1) \text { and } r_{\ell}=r_{1} \prod_{t=2}^{\ell} d_{t}=2^{-\ell}\left(1+O\left(j n^{-1 / 3}\right)\right)=2^{-\ell}\left(1+O\left(n^{-1 / 12}\right)\right) .
$$

Finally, from $m>\log n$ it follows that $j$ tends to infinity and thus $\sum_{\ell=1}^{j} r_{\ell}=1-o(1)$. Consequently, $\sum_{\ell=j}^{k} r_{\ell}=o(1)$.

It remains to show that we can modify our construction so that it has size $(2 / 3+\varepsilon)\left(\begin{array}{c}n \\ n / 2\end{array}\right)$ for some fixed $\varepsilon>0$. In order to do so we replace some of the set systems in the canonical partition. First note that for any $\beta>0$ the $\operatorname{sum} \sum_{i=n / 4-\beta n^{1 / 2}}^{n / 4}\left(\begin{array}{c}n / 2 \\ i\end{array}\right)^{2}$ is a positive fraction of $\sum_{i=0}^{n / 4}\left(\begin{array}{c}n / 2 \\ i\end{array}\right)^{2}$. Thus we will be done if for each $i$ with $n / 4-\beta n^{1 / 2} \leq i \leq n / 4$ we can replace the set systems of the canonical partition that contain $i$-sets with other $i$-uniform set systems $\mathcal{H}_{1}^{i}, \mathcal{H}_{2}^{i}, \ldots, \mathcal{H}_{s_{i}}^{i}$ such that $\sum_{t=1}^{s_{i}}\left|\mathcal{H}_{t}^{i}\right|^{2}$ is at least $(1 / 3+\varepsilon)\left(\begin{array}{c}n / 2 \\ i\end{array}\right)^{2}$ for some positive $\varepsilon$.

Frankl and Füredi considered in [7] the following pair of $i$-uniform intersecting set systems on a base set $Y$ : let $Y$ be equipartitioned into $Y_{1} \cup Y_{2}$ and define

$$
\begin{gathered}
\mathcal{G}_{1}^{i}=\left\{G \in\left(\begin{array}{c}
Y \\
i
\end{array}\right):\left|Y_{1} \cap G\right|>\left|Y_{1}\right| / 2\right\}, \\
\mathcal{G}_{2}^{i}=\left\{G \in\left(\begin{array}{c}
Y \\
i
\end{array}\right) \backslash \mathcal{G}_{1}:\left|Y_{2} \cap G\right|>\left|Y_{2}\right| / 2\right\} .
\end{gathered}
$$

They observed that if $|Y|=2 i+o\left(i^{1 / 2}\right)$, then $\left|\mathcal{G}_{1}^{i} \cup \mathcal{G}_{2}^{i}\right|=(1-o(1))\left(\begin{array}{c}|Y| \\ i\end{array}\right)$ and that for any $\alpha>0$ there exists $\beta>0$ such that if $|Y| \leq 2 i+\beta i^{1 / 2}$, then $\left|\mathcal{G}_{1}^{i} \cup \mathcal{G}_{2}^{i}\right| \geq(1-\alpha)\left(\begin{array}{c}|Y| \\ i\end{array}\right)$.

Let us fix $0<\alpha<1 / 6$ and consider $\beta$ as above. We define a modified version of the canonical partition for a given set $Y$. We replace the set systems $\mathcal{Y}_{i, j}$ for all $\frac{|Y|}{2}-\frac{\beta}{2 \sqrt{2}}|Y|^{1 / 2} \leq i \leq \frac{|Y|}{2}$ and $j=1, \ldots,|Y|-2 i+1$ with $\mathcal{G}_{1}^{i}$ and $\mathcal{G}_{2}^{i}$. As $\left|\mathcal{G}_{1}^{i}\right|+\left|\mathcal{G}_{2}^{i}\right| \geq$ $(1-\alpha)\left(\begin{array}{c}n / 2 \\ i\end{array}\right)$, the ratio of $\left|\mathcal{G}_{1}^{i}\right|^{2}+\left|\mathcal{G}_{2}^{i}\right|^{2}$ and $\left(\begin{array}{c}n \\ i\end{array}\right)^{2}$ is at least $2\left(\frac{1-\alpha}{2}\right)^{2}=1 / 2-\alpha+\alpha^{2} / 2$ which is strictly larger than $1 / 3$ by choice of $\alpha$. 
Katona's proof that a 2-part Sperner set system can contain at most $\left(\begin{array}{c}n \\ \lceil n / 2\rceil\end{array}\right)$ sets used a theorem of Erdös [3] on the number of sets contained in the union of $k$ Sperner set systems. Our proofs of Theorem 2 and Remark 7 used Theorem 6, Kleitman's result on the size of the union of $k$ intersecting families. It seems natural to ask how large can the union of $k$ intersecting Sperner set systems be as the problem seems to be interesting on its own right and it might help establishing bounds on 2S2I-systems. Unfortunately, we were only able to determine the exact result in the very special case when $k=2$ and $n$ is odd. The result follows easily from the following theorem of Greene, Katona and Kleitman.

Theorem 10 (Greene, Katona, Kleitman [8]). If $\mathcal{F} \subseteq 2^{[n]}$ is an intersecting and Sperner set system, then the following inequality holds

$$
\sum_{F \in \mathcal{F},|F| \leq n / 2} \frac{1}{\left(\begin{array}{c}
n \\
|F|-1
\end{array}\right)}+\sum_{F \in \mathcal{F},|F|>n / 2} \frac{1}{\left(\begin{array}{c}
n \\
|F|
\end{array}\right)} \leq 1 .
$$

Corollary 11. Let $\mathcal{F}, \mathcal{G} \subseteq 2^{[n]}$ be intersecting Sperner set systems and $n=2 l+1$ an odd integer. Then we have $|\mathcal{F} \cup \mathcal{G}| \leq\left(\begin{array}{c}n \\ l+1\end{array}\right)+\left(\begin{array}{c}n \\ l+2\end{array}\right)$ and the inequality is sharp as shown by $\mathcal{F}=\left(\begin{array}{c}{[n]} \\ l+1\end{array}\right), \mathcal{G}=\left(\begin{array}{c}{[n]} \\ l+2\end{array}\right)$.

Proof. We may assume that $\mathcal{F}$ and $\mathcal{G}$ are disjoint. Let us add the inequality of Theorem 10 for both systems $\mathcal{F}$ and $\mathcal{G}$. The bigger the number of the summands, the greater the cardinality of $\mathcal{F}$, therefore we need to keep the summands as small as possible to obtain the greatest number of summands. The set size for which the summand is the smallest is $l+1$ and the second smallest summand is for set sizes $l$ and $l+2$. As by the disjointness of the systems the number of smallest summands is at most $\left(\begin{array}{c}n \\ l+1\end{array}\right)$, the result follows.

\section{1-part intersecting, 1-part Sperner systems}

In this section we study 1-part Sperner 1-part intersecting set systems and prove Theorem 4. In order to prove the result we need a further definition. We say that the set systems $\mathcal{F}$ and $\mathcal{G}$ are intersecting, cross-Sperner if both $\mathcal{F}$ and $\mathcal{G}$ are intersecting and there is no $F \in \mathcal{F}, G \in \mathcal{G}$ with $F \subset G$ or $G \subset F$. We will prove the following theorem which can be of independent interest.

Theorem 12. Let $\mathcal{F}, \mathcal{G} \subset 2^{[n]}$ be a pair of cross-Sperner, intersecting set systems. Then we have

$$
|\mathcal{F}|+|\mathcal{G}| \leq 2^{n-1}
$$

and this bound is best possible.

One of our main tools will be the following special case of the Four Functions Theorem of Ahlswede and Daykin [1]. Let us write $\mathcal{A} \wedge \mathcal{B}=\{A \cap B: A \in \mathcal{A}, B \in \mathcal{B}\}$ and $\mathcal{A} \vee \mathcal{B}=\{A \cup B: A \in \mathcal{A}, B \in \mathcal{B}\}$. 
Theorem 13 (Ahlswede-Daykin, [1]). For any pair $\mathcal{A}, \mathcal{B}$ of set systems we have

$$
|\mathcal{A}||\mathcal{B}| \leq|\mathcal{A} \wedge \mathcal{B}||\mathcal{A} \vee \mathcal{B}|
$$

The other result we will use in our argument is due to Marica and Schönheim [12] and involves the difference set system $\Delta(\mathcal{F})=\left\{F \backslash F^{\prime}: F, F^{\prime} \in \mathcal{F}\right\}$.

Theorem 14 (Marica - Schönheim [12]). For any set system $\mathcal{F}$ we have $|\Delta(\mathcal{F})| \geq|\mathcal{F}|$.

Corollary 15. Let $\mathcal{D}$ be a downward closed set system and let $\mathcal{F}$ be an intersecting subsystem of $\mathcal{D}$. Then the inequality $2|\mathcal{F}| \leq|\mathcal{D}|$ holds.

Proof. As $\mathcal{D}$ is downward closed and $\mathcal{F} \subset \mathcal{D}$, it follows that $\Delta(\mathcal{F}) \subset \mathcal{D}$. Furthermore, as $\mathcal{F}$ is intersecting, we have $\mathcal{F} \cap \Delta(\mathcal{F})=\emptyset$ and thus we are done by Theorem 14 .

Proof of Theorem 12. Let us begin with defining the following four set systems

$$
\begin{array}{ll}
\mathcal{U}=\{U \subseteq[n]: \exists H \in \mathcal{F} \cup \mathcal{G} \text { such that } H \subseteq U\}, & \mathcal{U}^{\prime}=\mathcal{U} \backslash(\mathcal{F} \cup \mathcal{G}), \\
\mathcal{D}=\{D \subseteq[n]: \exists H \in \mathcal{F} \cup \mathcal{G} \text { such that } \quad D \subseteq H\}, & \mathcal{D}^{\prime}=\mathcal{D} \backslash(\mathcal{F} \cup \mathcal{G}) .
\end{array}
$$

Clearly, $\mathcal{D}^{\prime \prime}=\left\{D^{\prime}: \exists F \in \mathcal{F}\right.$ such that $\left.D^{\prime} \subset F\right\}$ is downward closed (and, by definition, $\mathcal{F} \subset \mathcal{D}^{\prime \prime}$ ), hence by Corollary 15 we have $2|\mathcal{F}| \leq\left|\mathcal{D}^{\prime \prime}\right|$. Moreover by the cross-Sperner property, we have $\left(\mathcal{D}^{\prime \prime} \backslash \mathcal{F}\right) \cap \mathcal{G}=\emptyset$, and therefore we have $\mathcal{D}^{\prime \prime} \backslash \mathcal{F} \subset \mathcal{D}^{\prime}$. Consequently $|\mathcal{F}| \leq\left|\mathcal{D}^{\prime}\right|$ and, by symmetry, $|\mathcal{G}| \leq\left|\mathcal{D}^{\prime}\right|$ also holds.

Note that $\mathcal{F} \wedge \mathcal{G} \subset \mathcal{D}^{\prime}$. Indeed, $F \cap G \in \mathcal{D}$ by definition and $F \cap G \in \mathcal{F}$ (or $F \cap G \in \mathcal{G}$ ) would contradict the cross-Sperner property. Similarly, we obtain that $\mathcal{F} \vee \mathcal{G} \subset \mathcal{U}^{\prime}$ and it is easy to see that the cross-Sperner property implies that $\mathcal{U}^{\prime} \cap \mathcal{D}^{\prime}=\emptyset$ and thus the four systems $\mathcal{F}, \mathcal{G}, \mathcal{U}^{\prime}, \mathcal{D}^{\prime}$ are pairwise disjoint.

Now suppose as a contradiction that $|\mathcal{F}|+|\mathcal{G}|>2^{n-1}$ and thus $\left|\mathcal{U}^{\prime}\right|+\left|\mathcal{D}^{\prime}\right|<2^{n-1}$. By $|\mathcal{F}|,|\mathcal{G}| \leq\left|\mathcal{D}^{\prime}\right|$ we obtain that $\left|\mathcal{U}^{\prime}\right|<|\mathcal{F}|,|\mathcal{G}|$ and thus using Theorem 13 we have

$$
\left|\mathcal{U}^{\prime}\right|\left|\mathcal{D}^{\prime}\right|<|\mathcal{F}||\mathcal{G}| \leq|\mathcal{F} \wedge \mathcal{G}||\mathcal{F} \vee \mathcal{G}| \leq\left|\mathcal{U}^{\prime}\right|\left|\mathcal{D}^{\prime}\right|
$$

a contradiction.

Finally, let us mention some pairs of set systems for which the sum of their sizes equals $2^{n-1}$. Any maximum intersecting system $\mathcal{F}$ with $\mathcal{G}$ the empty set system is extremal, just as the pair $\mathcal{F}_{1}=\{F \subset[n]: 1 \in F, 2 \notin F\}, \mathcal{G}_{1}=\{G \subset[n]: 1 \notin G, 2 \in G\}$. Furthermore, for any $k \geq n / 2$ the pair $\mathcal{F}_{k}=\{F \subset[n]: 1 \in F,|F| \leq k\}, \mathcal{G}_{k}=\{G \subset[n]: 1 \notin G,|G| \geq k\}$ has the required property, too.

Proof of Theorem 4. First let us consider any pair of maximal intersecting systems $\mathcal{A} \subseteq$ $2^{X_{1}}, \mathcal{B} \subseteq 2^{X_{2}}$. Clearly, the set system $\mathcal{F}=\mathcal{A} \times \mathcal{B}$ is a 1 I1S-system as any pair of sets $F_{1}, F_{2} \in \mathcal{F}$ intersect both in $X_{1}$ and in $X_{2}$. This shows that a maximum 1I1S-system contains at least $2^{|X|-2}$ sets.

To obtain the upper bound of the theorem let $\mathcal{F}$ be any 1I1S-system. For any $A \subseteq X_{1}$ let $\bar{A}$ denote $X_{1} \backslash A$. By definition, both $\mathcal{F}_{A}$ and $\mathcal{F}_{\bar{A}}$ are intersecting systems, and no element of the first can contain any element of the second (and vice versa). In other words they form a pair of intersecting, cross-Sperner systems. Due to Theorem 12 we have $\left|\mathcal{F}_{A}\right|+\left|\mathcal{F}_{\bar{A}}\right| \leq 2^{\left|X_{2}\right|-1}$. The number of pairs $A, \bar{A}$ is $2^{\left|X_{1}\right|-1}$ therefore we have $|\mathcal{F}| \leq$ $2^{|X|-2}$. 


\section{References}

[1] R. Ahlswede, D. Daykin, An inequality for the weights of two families of sets, their unions and intersections, Probability Theory and Related Fields, 43 (1978), $183-185$.

[2] H. Aydinian, É. Czabarka, P.L. Erdős, L.A. SzÉkely, A tour of M-part L-Sperner families, J. Comb. Theory Ser. A, 118 (2011), 702-725.

[3] P. Erdős, On a lemma of Littlewood and Offord, Bull. Amer. Math. Soc., 51 (1945), 898-902.

[4] P. Erdős, C. Ko, R. RAdo, Intersection theorems for systems of finite sets, Quart. J. Math. Oxford, 12 (1961), 313-318.

[5] P.L. ERdős, G.O.H. Katona, Convex hulls of more-part Sperner families, Graphs and Combinatorics 2 (1986), 123-134.

[6] P.L. Erdős, G.O.H. Katona, All maximum 2-part Sperner families, J. Comb. Theory Ser. A, 43 (1986), 58-69.

[7] P. Frankl and Z. Füredi, Extremal problems concerning Kneser graphs, J. Comb. Theory, Ser. B, 40 (1986), 270-284.

[8] C. Greene, G.O.H. Katona, D.J. Kleitman, Extensions of the Erdős-Ko-Rado theorem, SIAM 55 (1976) 1-8.

[9] G.O.H. Katona, On a conjecture of Erdős and a stronger form of Sperner's theorem, Studia Sci. Math. Hung. 1 (1966), 59-63.

[10] D.J. Kleitman, Families of non-disjoint subsets, J. Comb. Theory, 1 1966, 153-155.

[11] D.J. Kleitman, On a lemma of Littlewood and Offord on the distribution of certain sums, Math. Z. 90 (1965), 251-259.

[12] J. Marica, J. Schönheim, Differences of sets and a problem of Graham, Can. Math. Bull. 12 (1969), 635-637.

[13] E. Sperner, Ein Satz über Untermenge einer endlichen Menge, Math Z., 27 (1928) $544-548$. 